PROCEEDINGS OF THE

AMERICAN MATHEMATICAL SOCIETY

Volume 136, Number 9, September 2008, Pages 3159-3162

S 0002-9939(08)09272-1

Article electronically published on March 11, 2008

\title{
SEPARATION OF SPECTRA FOR BLOCK TRIANGLES
}

\author{
ROBIN HARTE AND CORA STACK
}

(Communicated by Joseph A. Ball)

\begin{abstract}
Separation of the spectra of the diagonal elements of a block triangle corresponds to comparison with its fundamental projection.
\end{abstract}

Suppose

$$
G=\left(\begin{array}{cc}
A & M \\
N & B
\end{array}\right) \equiv\left\{\left(\begin{array}{cc}
a & m \\
n & b
\end{array}\right):(a, m, n, b) \in A \times M \times N \times B\right\}
$$

is a Banach algebra with block structure: thus $[3] A$ and $B$ are Banach algebras with identities and $M$ and $N$ are bimodules over $A$ and $B$ and everything is explained by formal matrix multiplication. An upper triangle in $G$ is an element of the form

$$
T=\left(\begin{array}{cc}
a & m \\
0 & b
\end{array}\right)
$$

It is well known [2, [3], that of the three spectra

$$
\sigma_{G}(T), \sigma_{A}(a), \sigma_{B}(b),
$$

each is contained in the union of the other two: this extends more generally to "spectral triangles" 4. Disjointness between the spectra of $a \in A$ and $b \in B$, or significant subsets of them, has consequences expressible 3 in terms of a comparison between the operators

$$
S=\left(\begin{array}{cc}
a & a m-m b \\
0 & b
\end{array}\right), P=\left(\begin{array}{cc}
1 & m \\
0 & 0
\end{array}\right) .
$$

1. Theorem. If $f \in \operatorname{Holo}(\sigma(a) \cup \sigma(b))$ is holomorphic near the spectra of $a \in A$ and $b \in B$, then

$$
f\left(\begin{array}{cc}
a & a m-m b \\
0 & b
\end{array}\right)=\left(\begin{array}{cc}
f(a) & f(a) m-m f(b) \\
0 & f(b)
\end{array}\right) .
$$

In particular if and only if $S$ has disjoint diagonal spectra

$$
\sigma_{A}(a) \cap \sigma_{B}(b)=\emptyset,
$$

then $P$ is a holomorphic function of $S$ :

$$
P=f(S) \text { with } f \in \text { Holo } \sigma(S) \text {. }
$$

Received by the editors January 30, 2007, and, in revised form, June 14, 2007.

2000 Mathematics Subject Classification. Primary 47A10.

Key words and phrases. Banach algebra, separation of spectra, block matrices. 
Proof. We are writing $f \in \operatorname{Holo}(K)$, for subsets $K \subseteq \mathbf{C}$, to mean that $f: D_{f} \rightarrow \mathbf{C}$ is defined and holomorphic on some neighbourhood $D_{f} \in \operatorname{Nbd}(K)$. Generally,

$$
f\left(\begin{array}{cc}
a & m \\
0 & b
\end{array}\right)=\left(\begin{array}{cc}
f(a) & f^{\wedge}(a ; m ; b) \\
0 & f(b)
\end{array}\right)
$$

with

$$
f^{\wedge}(a ; m ; b)=\frac{1}{2 \pi i} \oint_{\sigma(a) \cup \sigma(b)} f(z)(z-a)^{-1} m(z-b)^{-1} d z ;
$$

now replace $m$ by $a m-m b=m(z-b)-(z-a) m$ for (1.1). For (1.3) take

$$
f=\chi_{K} \text { with } K=\sigma(a) \subseteq \sigma(a) \cup \sigma(b) ;
$$

conversely if (1.3) holds, then

$$
\sigma_{A}(a) \subseteq f^{-1}(1), \sigma_{B}(b) \subseteq f^{-1}(0)
$$

are necessarily disjoint.

If in particular $m=0$, then $S=T$, as in Theorem 1 of 3 .

If we write [3], for $f \in \operatorname{Holo}(U, X)$ on open $U \subseteq \mathbf{C}$,

$$
\Delta f(\alpha, \beta)=\delta_{\alpha} f(\beta)=\delta_{\beta} f(\alpha)= \begin{cases}(f(\beta)-f(\alpha)) /(\beta-\alpha) & \beta \neq \alpha \\ f^{\prime}(\alpha) & \beta=\alpha\end{cases}
$$

then if $\sigma(a) \cup \sigma(b) \subseteq U$, we can write

$$
f^{\wedge}(a ; m ; b)=\Delta f\left(L_{a}, R_{b}\right)(m),
$$

at least if $\exists\left(L_{a}-R_{b}\right)^{-1} \in B(M)$. At another extreme, if $B=A$,

$$
f^{\wedge}(a ; m ; a)=d_{a} f(m)
$$

can be interpreted as a Fréchet differential.

If the projection $P$ is a holomorphic function of the operator $S$, then it lies in the double commutant of $S$ in $G$ :

2. Theorem. If $L_{a}-R_{b} \in B(M)$ and $L_{b}-R_{a} \in B(N)$ are left invertible, then we can factorize

$$
L_{S}-R_{S}=\mathbf{W}\left(L_{P}-R_{P}\right) \text { with } \mathbf{W} \in B(G) .
$$

Proof. Replacing $2 \times 2$ matrices with $4 \times 1$ columns, write

$$
\begin{aligned}
\left(L_{S}-R_{S}\right)^{\wedge} & =\left(\begin{array}{cccc}
L_{a}-R_{a} & 0 & L_{a m-m b} & 0 \\
-R_{a m-m b} & L_{a}-R_{b} & 0 & L_{a m-m b} \\
0 & 0 & L_{b}-R_{a} & 0 \\
0 & 0 & -R_{a m-m b} & L_{b}-R_{b}
\end{array}\right), \\
\left(L_{P}-R_{P}\right)^{\wedge} & =\left(\begin{array}{cccc}
0 & 0 & L_{m} & 0 \\
-R_{m} & I & 0 & L_{m} \\
0 & 0 & -I & 0 \\
0 & 0 & -R_{m} & 0
\end{array}\right) ;
\end{aligned}
$$


now with

$$
U\left(L_{a}-R_{b}\right)=I=V\left(L_{b}-R_{a}\right)
$$

take

$$
\mathbf{W}^{\wedge}=\left(\begin{array}{cccc}
0 & 0 & L_{m} V & 0 \\
-U R_{m} & U & U\left(L_{m}+R_{m}\right) V & U L_{m} \\
0 & 0 & -V & 0 \\
0 & 0 & -R_{m} V & 0
\end{array}\right)
$$

Sufficient for the left invertibility of $L_{a}-R_{b}$ and $L_{b}-R_{a}$ is [1] a weaker spectral disjointness:

$$
\sigma_{A}^{l e f t}(a) \cap \sigma_{B}^{r i g h t}(b)=\emptyset=\sigma_{A}^{r i g h t}(a) \cap \sigma_{B}^{l e f t}(b) ;
$$

this disjointness is in turn necessary if [3] the algebra $G$ is for example "ultraprime". It is sufficient for the double commutant condition that the operator $L_{a}-R_{b}$ is one-one:

\section{Theorem. If}

$$
\left(L_{a}-R_{b}\right)^{-1}(0)=\{0\} \subseteq M
$$

then

$$
\left(L_{S}-R_{S}\right)^{-1}(0) \subseteq\left(L_{P}-R_{P}\right)^{-1}(0) \subseteq G
$$

Proof. Generally,

$$
\begin{aligned}
\left(L_{S}\right. & \left.-R_{S}\right)\left(\begin{array}{cc}
a^{\prime} & m^{\prime} \\
n^{\prime} & b^{\prime}
\end{array}\right)=O \Longleftrightarrow a^{\prime} a-a a^{\prime}=b^{\prime} b-b b^{\prime}=n^{\prime}=0 \\
& =a m^{\prime}-m^{\prime} b-a^{\prime}(a m-m b)+(a m-m b) b^{\prime} \\
& \Longrightarrow n^{\prime}=m n^{\prime}=0=\left(L_{a}-R_{b}\right)\left(m^{\prime}-a^{\prime} m+m b^{\prime}\right) \\
& \Longrightarrow n^{\prime}=m n^{\prime}=0=m^{\prime}-a^{\prime} m+m b^{\prime},
\end{aligned}
$$

giving if $\left(L_{a}-R_{b}\right)^{-1}(0)=\{0\}$,

$$
\left(L_{P}-R_{P}\right)\left(\begin{array}{cc}
a^{\prime} & m^{\prime} \\
n^{\prime} & b^{\prime}
\end{array}\right)=O .
$$

The condition (3.2) says precisely that the commutant of $S$ is a subalgebra of the commutant of $P$, or equivalently that $P$ is in the double commutant of $S$. Sufficient ([3], Theorem 2) is that the left approximate point spectrum of $a \in A$ is disjoint from the right approximate point spectrum of $b \in B$. If the algebra $G$ is "prime", then it is necessary that the corresponding point spectra are disjoint.

\section{REFERENCES}

[1] R. E. Harte, Invertibility and singularity for bounded linear operators, Marcel Dekker, 1988. MR0920812 (89d:47001)

[2] R. E. Harte, Invertibility and singularity of operator matrices, Proc. Royal Irish Acad. 88A (1988), 103-118. MR986217(90a:15019) 
[3] R. E. Harte, Block diagonalization in Banach algebras, Proc. Amer. Math. Soc. 129 (2000), 181-190. MR 1784022 (2001k:47013)

[4] R. E. Harte and C. M. Stack, Invertibility for spectral triangles, Operators and Matrices 1 (2007), 445-453.

School of Mathematics, Trinity College, Dublin, Ireland

E-mail address: rharte@maths.tcd.ie

Department of Mathematics, Institute of Technology at Tallaght, Dublin, Ireland

E-mail address: cora.stack@ittdublin.ie 